\title{
A paracentesis to save time and money with re-bubbling after descemets membrane endothelial keratoplasty
}

Eye (2018) 32, 238-239; doi:10.1038/eye.2017.189; published online 1 September 2017

Descemet's membrane endothelial keratoplasty (DMEK) is becoming more popular among corneal surgeons for management of endothelial diseases. The benefits of endothelial keratoplasties include lower rate of rejection, rapid visual recovery, and less surgically induced astigmatism. ${ }^{1}$ The frequently encountered frustration with DMEK is partial graft detachment in the early postoperative period, which varies in several studies, with one multicentre study reporting the occurrence rate to be as high as $34.6 \% .^{2}$ Typically,

partial graft detachments are treated by bringing the patients back to theatre for a re-bubbling procedure. This results in additional costs of theatre utilisation, surgeon time, and staff time.

In this article, we describe the creation of a paracentesis on 5 o'clock (for right-handed surgeons) or 7 o'clock (for left-handed surgeons) at the limbus with a $15^{\circ}$ blade before completing the DMEK procedure in the operating theatre as illustrated in Figure 1. The creation of this paracentesis can be in addition to or as a part of the required DMEK surgery paracentesis incisions (based on whether the surgeon is sitting superiorly or temporally). This allows the surgeon to re-bubble the anterior chamber using a simple insulin syringe with a bent needle or a $2 \mathrm{ml}$ syringe with a cannula on the slit lamp under topical anaesthesia using the right hand after instillation of G. Povidone Iodine 5\% during the clinic follow-up. The patient is prescribed topical antibiotics for a week and topical steroids as per the surgeon's protocol after the clinic re-bubbling procedure.

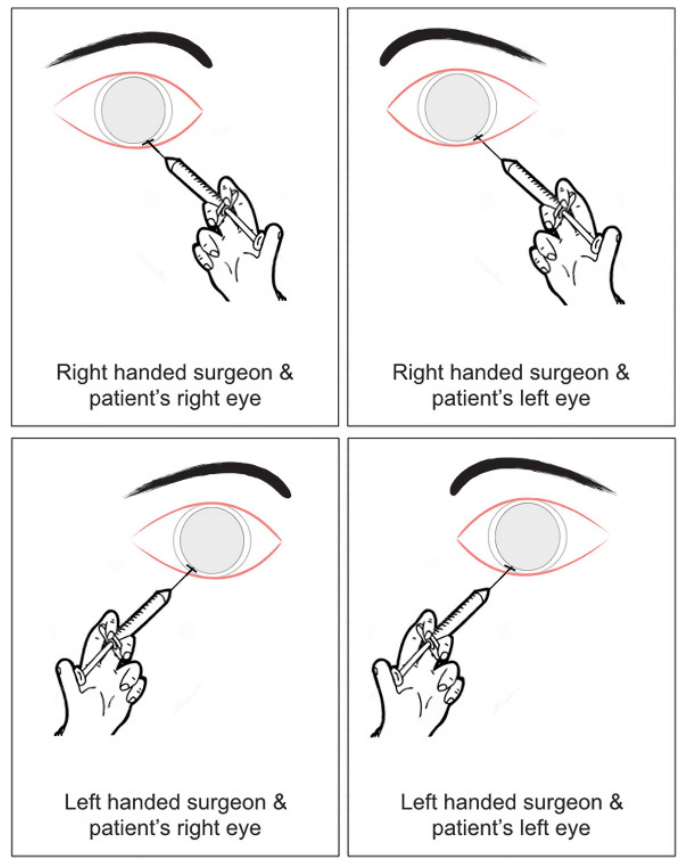

Figure 1 This figure shows the use of 5 o'clock and 7 o'clock paracentesis, respectively, for right- and lefthanded surgeons, on a slit lamp for re-bubbling partial detachment of DMEK grafts.

This paracentesis incision avoids the need for bringing the patient back to the operating theatre, thus reducing the costs and time. This paracentesis can be used up to 2 weeks postoperatively on the slit lamp. The paracentesis is created at 5 o'clock with the assumption that the surgeon is right-handed, and can be modified depending on hand dominance (Figure 1). Using this new paracentesis in all our DMEK patients, we have been successful in 100\% avoidance of re-admission to the theatre and cost reductions for re-bubbling procedures. 


\section{Conflict of interest}

The authors declare no conflict of interest.

\section{References}

1 Nanavaty MA, Wang X, Shortt AJ. Endothelial keratoplasty versus penetrating keratoplasty for Fuchs endothelial dystrophy. Cochrane Database Syst Rev 2014; (2): CD008420.

2 Monnereau C, Quilendrino R, Dapena I, Liarakos VS, Alfonso JF, Arnalich-Montiel F et al. Multicenter study of descemet membrane endothelial keratoplasty: first case series of 18 surgeons. JAMA Ophthalmol 2014; 132(10): 1192-1198.
FM Chew ${ }^{1}$, K Teeluck ${ }^{2}$ and MA Nanavaty ${ }^{1,2}$

${ }^{1}$ Sussex Eye Hospital, Brighton and Sussex University Hospitals NHS Trust, Brighton, UK

${ }^{2}$ Brighton \& Sussex Medical School, University of Sussex, Falmer, Brighton, UK

Correspondence: MA Nanavaty, Sussex Eye Hospital, Brighton and Sussex University Hospitals NHS Trust,

Brighton BN2 5BF, UK Tel: 01273606126 ;

Fax: 00441273664610 .

E-mail: mayank.nanavaty@bsuh.nhs.uk 